\title{
A Compact Coupled-Fed Loop Antenna for Mobile LTE Smartphones
}

\author{
Ping Wang $\mathbb{D}^{1},{ }^{1}$ Yu Shao, ${ }^{1}$ Dong Huang, ${ }^{2}$ and Muhammad Abdul Basit ${ }^{3}$ \\ ${ }^{1}$ School of Communications and Information Engineering, Chongqing University of Posts and Telecommunications, \\ No. 2, Chongwen Road, Nan'an District, Chongqing 400065, China \\ ${ }^{2}$ Chongqing Institute of Green and Intelligent Technology, Chinese Academy of Sciences, Beijing 400714, China \\ ${ }^{3}$ Electronics Department, University of Engineering \& Technology Taxila Sub-Campus, Chakwal, Pakistan
}

Correspondence should be addressed to Ping Wang; wp@cqupt.edu.cn

Received 23 September 2017; Accepted 21 November 2017; Published 11 March 2018

Academic Editor: Shih Yuan Chen

Copyright (c) 2018 Ping Wang et al. This is an open access article distributed under the Creative Commons Attribution License, which permits unrestricted use, distribution, and reproduction in any medium, provided the original work is properly cited.

\begin{abstract}
A coupled-fed loop antenna with Octa-bands operation for long-term evolution (LTE) smartphones is proposed in this paper. The antenna occupies a nonground space of only $6.5 \mathrm{~mm} \times 72 \mathrm{~mm}$, and two wide-band operations can be achieved by exploiting the multimode characteristics of loop antenna and using high-pass matching circuits. In low band, the LTE700/GSM850/900 operation is achieved by the loop mode of $0.5 \lambda$ and matching chip capacitor which generates a dual-resonance mode at $0.74 \mathrm{GHz}$ and $0.9 \mathrm{GHz}$. In high band, the $1 \lambda$ mode, the $1.5 \lambda$ mode, and the $2 \lambda$ mode of the entire loop are combined with the $0.5 \lambda$ mode of the left-side coupling loop to cover the DCS1800/PCS1900/UMTS 2100/LTE 2300/2500 operation bands. The measured average realized gains and efficiencies are approximately $1.56 \mathrm{dBi} / 2.38 \mathrm{dBi}$ and $55.3 \% / 63.6 \%$ in the two bands, and a good radiation pattern is achieved as well.
\end{abstract}

\section{Introduction}

Mobile long-term evolution (LTE) smartphone has become an essential equipment for daily life bringing a lot of convenience due to its integrated multifunction services, for example, mobile communications, surfing the Internet, GPS location, and e-payment. The increasing application area will become more and more obvious in the future. As a critical component of mobile smartphones, the antennas with various configurations have been investigated widely to cover multiple frequency bands maturing 2G/3G/4G wireless communication services [1]. In addition, keeping in view the customer's demand for a large display screen and a narrow frame, the height of the nonground space for the handset antenna needs to be kept at a minimum. Generally, the height with less than $10 \mathrm{~mm}$ fulfills the design requirement of modern mobile phones [2]. So, designing a multiband/wideband mobile antenna with less nonground space is not only an essential requirement but also a major technical challenge.
The design configuration of handset antennas can be basically categorized into four distinct types: The first type uses coupled-fed monopole antenna configuration for wideband or multiband applications [2]. The second one uses reconfigurable technique to realize multiband operation [3]. The third one is the slot antenna configuration [4]. The fourth one includes the various types of loop antenna [5-8]. Because of both multiresonant mode and high-efficiency characteristics of the loop antenna, that is, the $0.5 \lambda$ monopole mode, the $1 \lambda$ dipole mode, the $1.5 \lambda$ higher order monopole mode, and the $2 \lambda$ higher order dipole mode [9], in which the dipole mode offer better user experience due to its balance characteristic [10], the loop antenna is first considered and developed for wideband or multiband antennas of mobile phones comparing to other types of configuration. However, generally, it is difficult to cover the $2 \mathrm{G} / 3 \mathrm{G} / 4 \mathrm{G}$ wireless communication bands completely using a single-loop structure [9]. In order to meet the bandwidth requirement, various hybrid composite structures were introduced to cover the full LTE band, including loop/monopole/dipole 


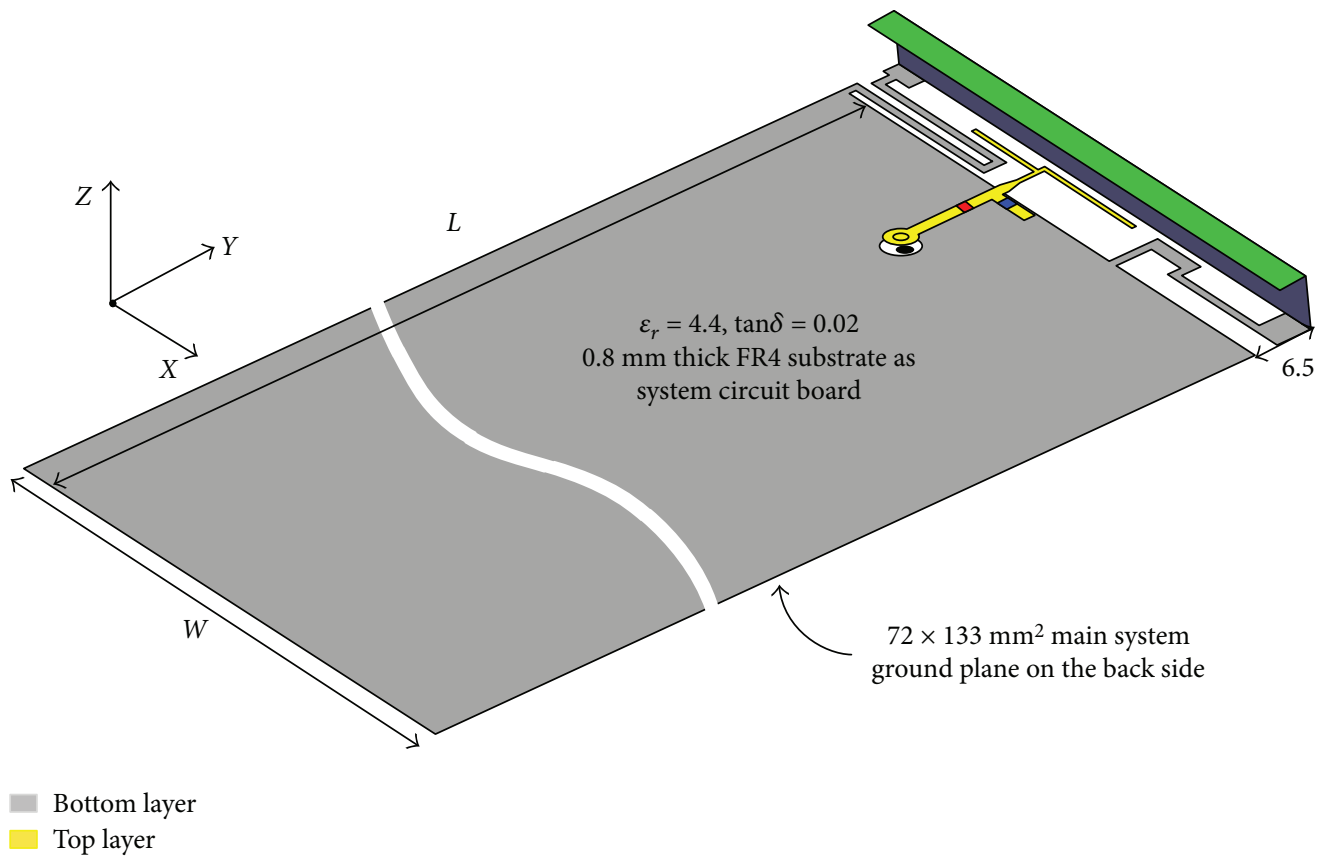

(a)

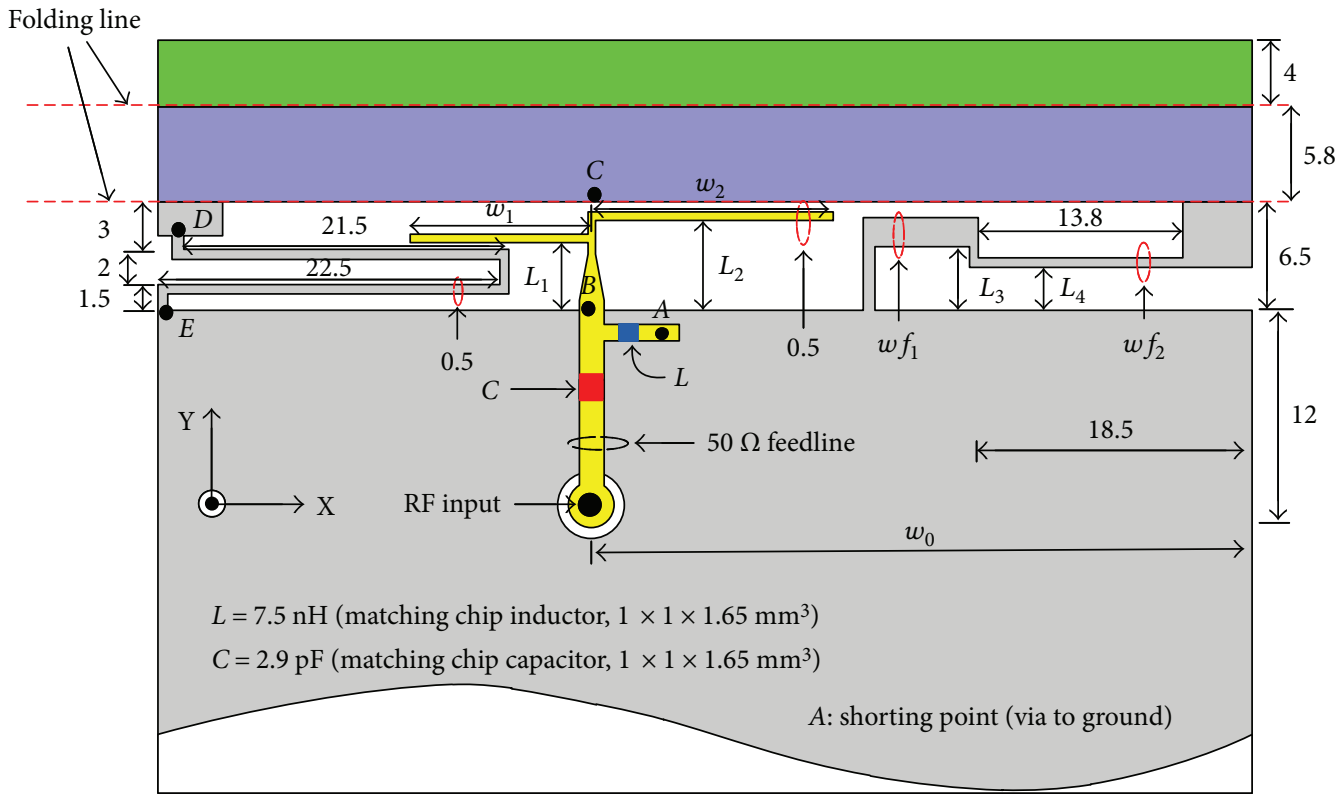

(b)

FIgURE 1: Geometry and dimensions of the proposed antenna. (a) Top view of the wideband antenna for Octa-band LTE/WWAN operation. (b) Enlargement dimensions of the metal pattern in the antenna area. $L=133.5, W=72, w_{1}=12, w_{2}=15.95, L_{1}=4.3, L_{2}=4.9, L_{3}=3.8$, $L_{4}=2.5, w f_{1}=1.7, w f_{2}=0.5$, and $w_{0}=43.5$. Unit: $\mathrm{mm}$.

antenna [10], loop/open-slot antenna [11], segmented dualloop antenna [12], and dual-loop metal-rimmed antenna [7]. The objective of the hybrid combination is to add more resonant modes and to enhance the bandwidth. Though the abovementioned methods are effective to realize lager bandwidths, it is difficult and challenging to maintain the full LTE band coverage if the height of nonground space continues to be reduced under the temptation of a large display screen and a narrow frame.
In this paper, an Octa-band loop antenna with two wide operating bands and a very small nonground height of only $6.5 \mathrm{~mm}$ is presented. The lower band has a bandwidth of $0.7 \mathrm{GHz}$ to $1.03 \mathrm{GHz}(0.33 \mathrm{GHz}$ or $38.2 \%$ bandwidth $)$ to cover the LTE700/GSM850/GSM900 systems, whereas the upper band from $1.7 \mathrm{GHz}$ to $2.69 \mathrm{GHz}(0.99 \mathrm{GHz}$ or $45.1 \%$ bandwidth) covers the DCS1800/PCS1900/UMTS/TD-SCDMA/ LTE2300/2500 systems. The performance of the antenna is studied using the ANSYS high-frequency structure simulator 


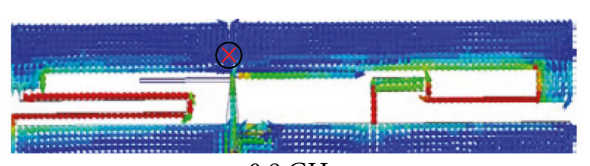

$0.9 \mathrm{GHz}$

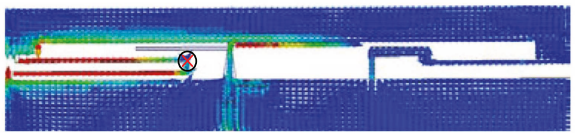

$2.1 \mathrm{GHz}$

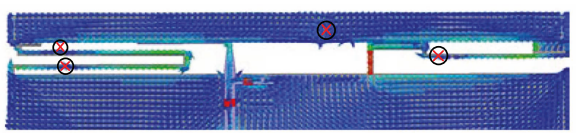

$2.64 \mathrm{GHz}$

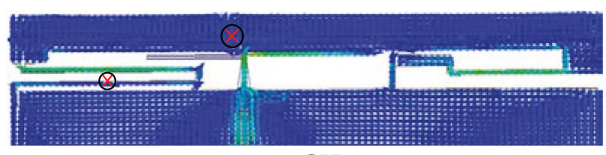

$1.8 \mathrm{GHz}$

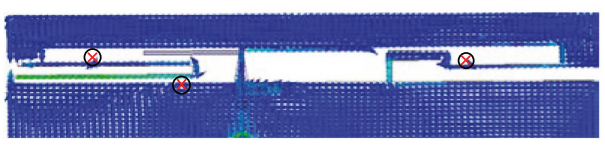

$2.3 \mathrm{GHz}$
Jsurf [A_per_m]

$1.0000 e+002$ $9.2857 e+001$ $8.5714 e+001$ $7.8571 e+001$ $7.1429 e+001$ $6.4286 e+001$ $5.7143 e+001$ $5.0000 e+001$ $4.2857 e+001$ $3.5714 e+001$ $2.8571 e+001$ $2.1429 e+001$ $1.4286 e+001$ $7.1429 e+000$ $0.0000 e+000$

$\otimes$ Current null

FIgURE 2: Vector current distribution of the proposed antenna at frequencies of $0.9 \mathrm{GHz}, 1.8 \mathrm{GHz}, 2.1 \mathrm{GHz}, 2.3 \mathrm{GHz}$, and $2.64 \mathrm{GHz}$.

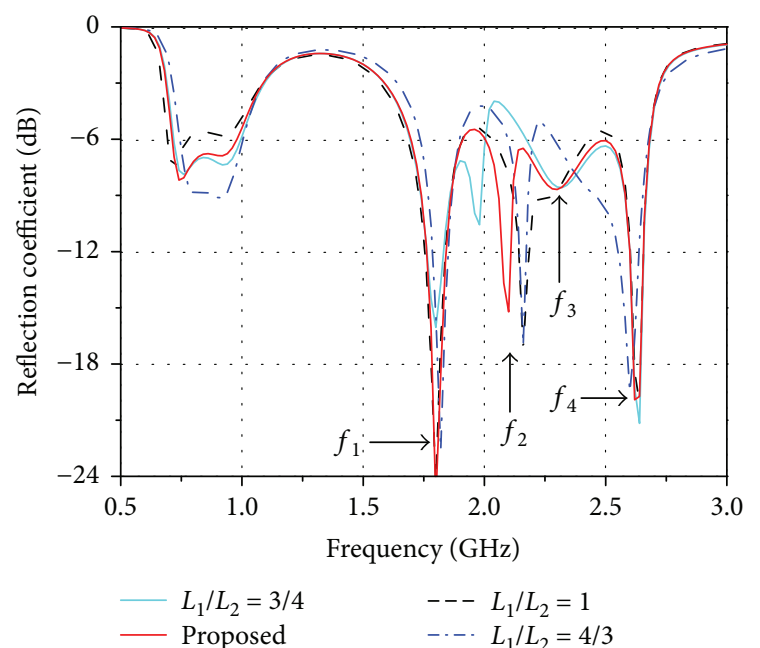

FIGURE 3: Simulated reflection coefficient for the proposed antenna as a function of the ratio between $L_{1}$ and $L_{2}$.

(HFSS). A prototype of the antenna is fabricated and measured to verify the design.

\section{Proposed Antenna Design}

2.1. Antenna Configuration. Figure 1(a) presents the geometry of the proposed coupled-fed loop antenna, while the planar metal enclosure of the antenna can be seen in Figure 1(b). The design employs a $0.8 \mathrm{~mm}$ FR4 substrate (dielectric constant $=4.4$, loss tangent $=0.02$ ) with an outer dimension of about $72 \mathrm{~mm} \times 139.5 \mathrm{~mm}$ as the circuit board of the smartphone. The size of the system ground plane, printed on the back layer of the substrate, was selected as $72 \mathrm{~mm} \times 133 \mathrm{~mm}$ so that $6.5 \mathrm{~mm} \times 72 \mathrm{~mm}$ ungrounded areas on the top side of the substrate can be utilized for the proposed antenna. A VIA hole is placed so that a connection can be made, for antenna testing, between the $50 \Omega$ feeding strip-line and the inner conductor of a $50 \Omega$ SMA connector. The external metal shell of the SMA connector is soldered to the system ground plane.

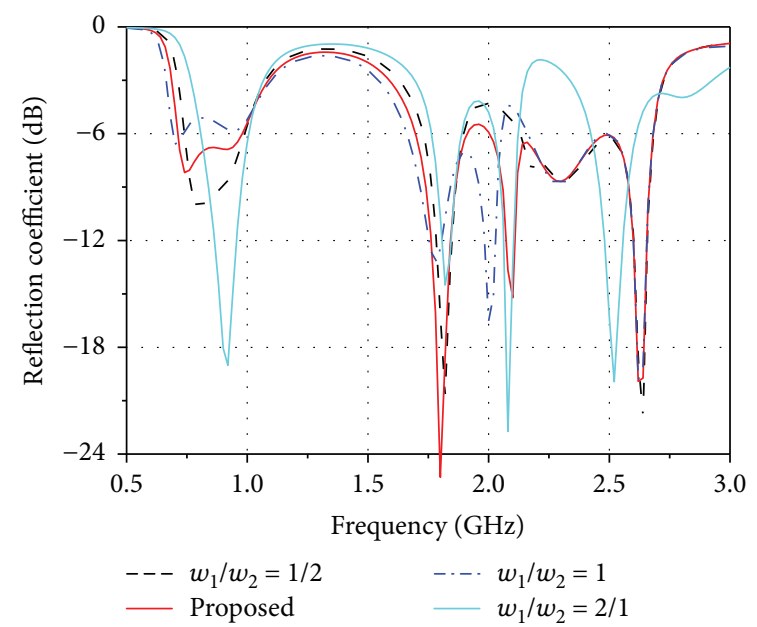

FIGURE 4: Effect of the ration $w_{1} / w_{2}$ on reflection coefficient.

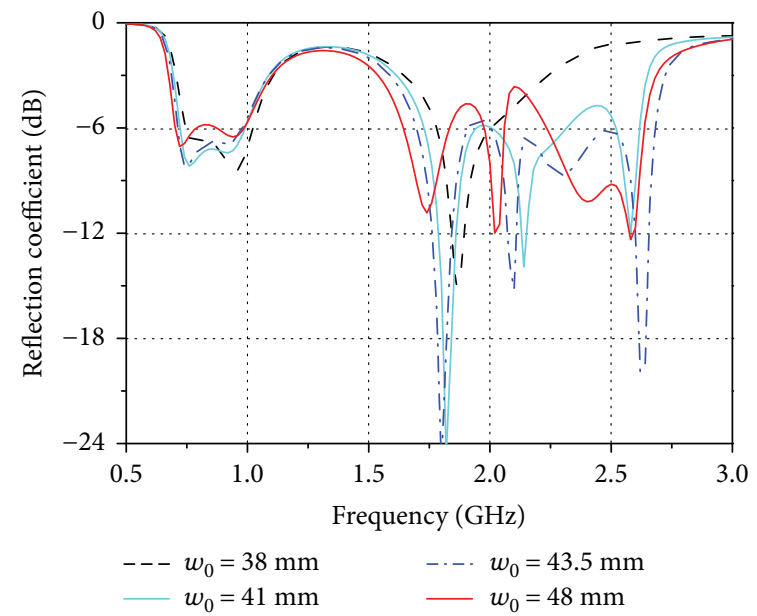

FIGURE 5: Simulated reflection coefficient for the proposed antenna as a function of $w_{0}$. 


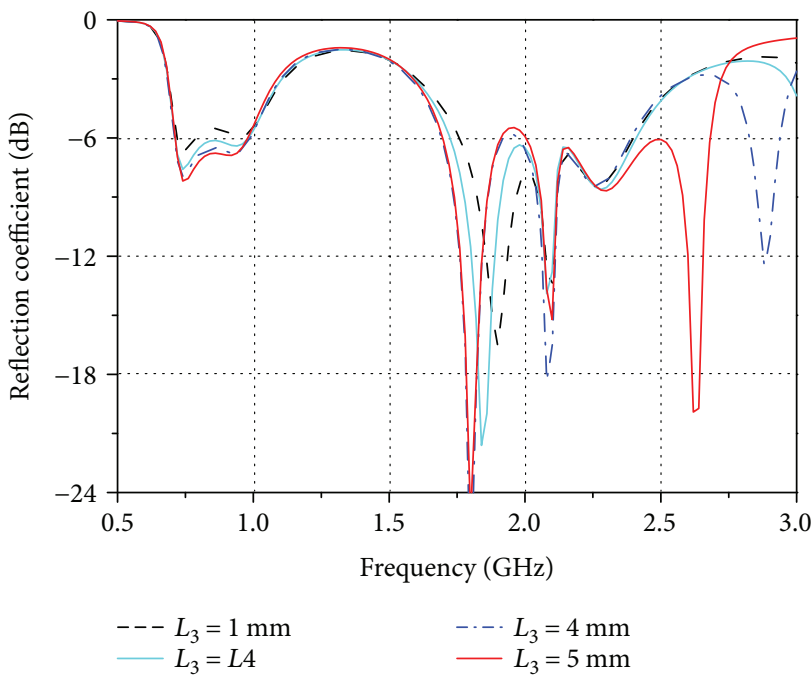

(a)

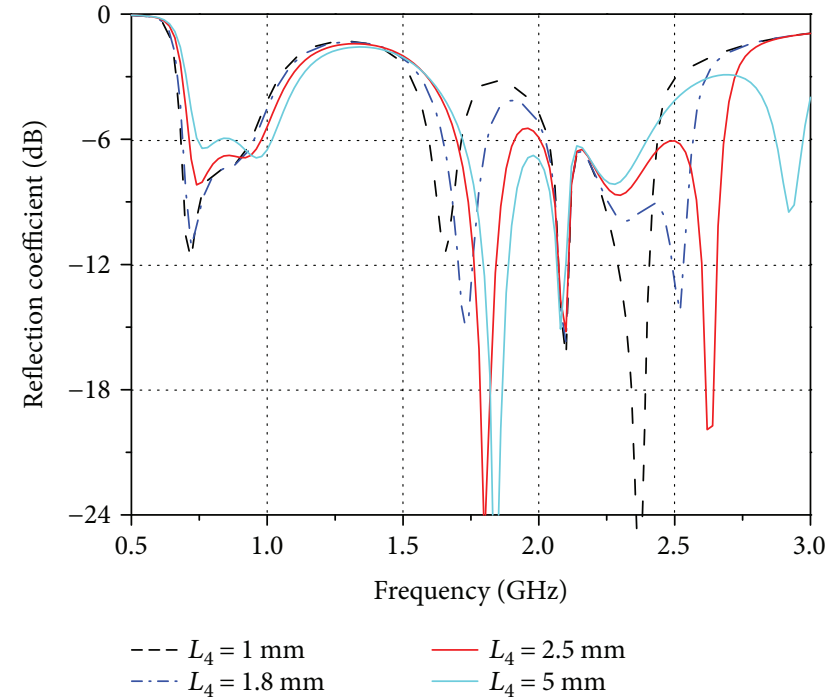

(b)

Figure 6: Simulated reflection coefficient for the proposed antenna as a function of (a) $L_{3}$ when the $L_{4}$ is kept unchanged and (b) $L_{4}$ when the $L_{3}$ is kept unchanged.

The loop antenna is composed of an unequal-arm Tshaped driven strip and a single-folded loop structure. It should be mentioned that the loop configuration differs from the reported loop antenna in the open literatures [5-12]. As seen in Figure 1(b), both front ends of the proposed loop antenna are connected to the system ground plane and are capacitively coupled by the T-shaped strip. A couple of folded microstrip lines are integrated with the loop on both sides to excite half-wavelength fundamental resonances at $0.9 \mathrm{GHz}$, which also resulted in smaller volume. Moreover, for bandwidth improvement purposes, a bent metallic plate is connected to the top edge of the loop. Figure 2 shows the simulated vector current distributions. It is clearly seen that the complete interior length of the loop is about $196 \mathrm{~mm}$ (equivalent to $0.5 \lambda$ at $0.9 \mathrm{GHz}$ ) generating the $0.5 \lambda, 1 \lambda, 1.5 \lambda$, and $2 \lambda$ modes at almost $0.9 \mathrm{GHz}$, 1.8 $\mathrm{GHz}, 2.3 \mathrm{GHz}$, and $2.64 \mathrm{GHz}$, respectively. Furthermore, the folded loop path of $\mathrm{B} \rightarrow \mathrm{C} \rightarrow \mathrm{D} \rightarrow \mathrm{E}$ on the left side of the ungrounded area is capacitively connected through a narrow coupling gap of $1.1 \mathrm{~mm}$ to excite the $0.5 \lambda$ modes at almost $2.1 \mathrm{GHz}$. The high-order resonance modes including $1 \lambda, 1.5 \lambda$, and $2 \lambda$ modes, and $0.5 \lambda$ modes of the left loop area are responsible for covering the DCS/PCS/ UMTS2100/LTE2300/2500 bands. An extra resonance was also generated at $0.74 \mathrm{GHz}$ by employing a $7.5 \mathrm{nH}$ chip inductor and a $2.9 \mathrm{pF}$ chip capacitor forming a high-pass circuit, which was combined with the fundamental mode of the $0.5 \lambda$ loop mode; both GSM850/900 can be covered. Lastly, two simulated bands ranging from $0.7 \mathrm{GHz}$ to $1.03 \mathrm{GHz}$ and $1.7 \mathrm{GHz}$ to $2.69 \mathrm{GHz}$ can be obtained to cover Octa bands.

2.2. Parametric Studies. To better understand the influence of the design parameters on the performance of the antenna, a parametric study was carried out.
Figure 3 shows the reflection coefficient variation over frequency for several values of the ratio between $L_{1}$ and $L_{2}$. The figure shows that variation of the ratio results in significant shift ups of the second resonance $\left(f_{2}\right)$, while hardly alters the first, third, and fourth resonances $\left(f_{1}, f_{3}\right.$, and $\left.f_{4}\right)$ in the upper frequency band. However, when the ration exceeds the value of 1 , the third mode is shifted up and is merged together with the fourth mode. Results have revealed that the best performance is obtained when $L_{1}=4.3 \mathrm{~mm}$ and $L_{2}=4.9 \mathrm{~mm}$.

Figure 4 demonstrates the impact of varying the ratio between $w_{1}$ and $w_{2}$ on the reflection coefficient of the antenna. It is clear from the figure that an increase in the ratio results in the separation of both modes in the lower band, and the second resonant mode $\left(f_{2}\right)$ in upper band shifts to a lower frequency, but barely affecting the first, third, and fourth modes. Furthermore, a continuous increase in the ratio leads to a point where only one mode is generated in the lower band, the third mode disappears, and the second resonant frequency increases contrarily in the upper band. The observations imply that better results can be achieved by carefully adjusting the ratios.

The effect of varying the parameter $w_{0}$ on the antenna reflection coefficient is shown in Figure 5. It is observed that the parameter $w_{0}$ has lager effect on the reflection coefficient in the upper band as compared to that in the lower band. Moreover, the number of resonant modes also increases with increasing $w_{0}$, that is, the number of the resonant modes increases from one to four by increasing the value of $w_{0}$ from $38 \mathrm{~mm}$ to $43.5 \mathrm{~mm}$. However, further increase in $w_{0}$ does not affect the number of the mode.

Figure 6 shows that the simulated reflection coefficient varies as a function of $L_{3}$ or $L_{4}$, where $w f_{1}$ is equal to $w f_{2}$ for choosing lager $L_{3}$. One can notice that the variation of $L_{3}$ and $L_{4}$ has stronger impact on upper bands than that on 


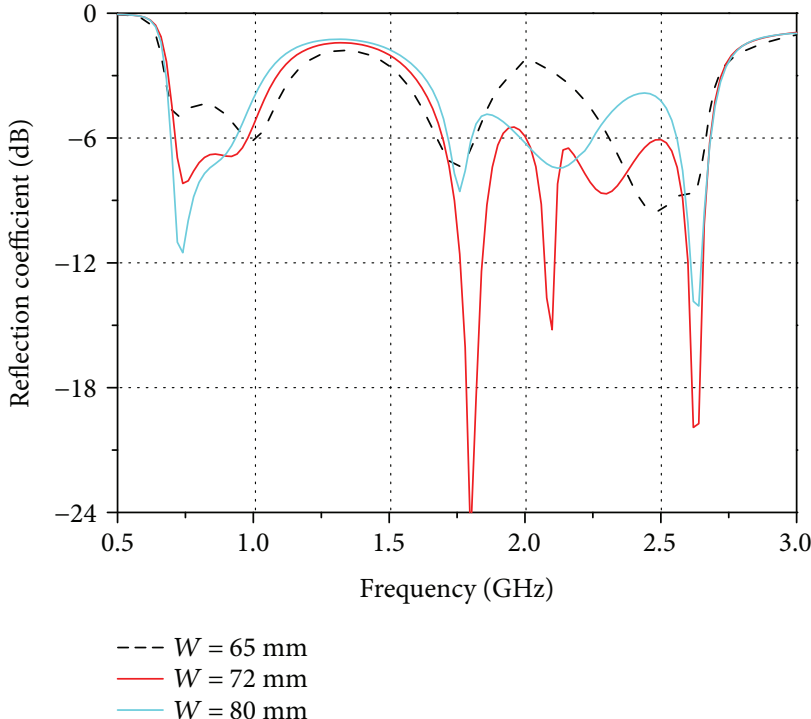

(a)

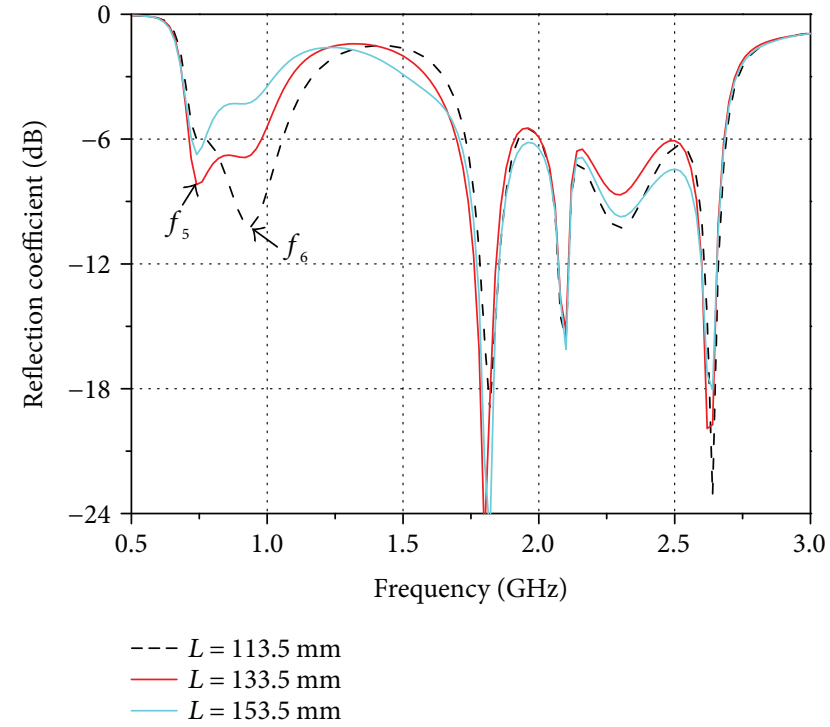

(b)

Figure 7: Effect of the ground plane on the reflection coefficient. (a) Width of the ground plane. (b) Length of the ground plane.

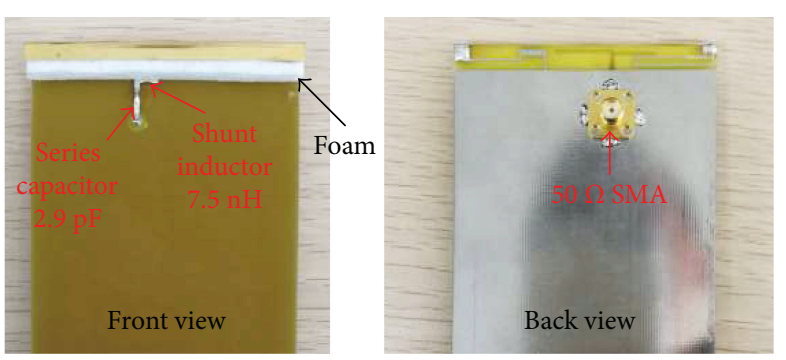

(a)

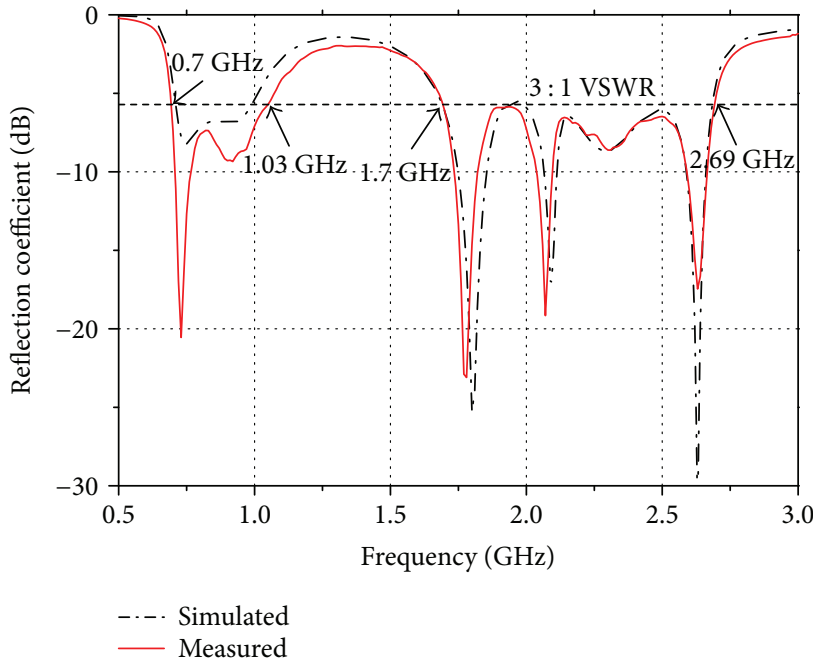

(b)

FIGURE 8: (a) Fabricated antenna and (b) comparison of simulated and measured reflection coefficient of the proposed antenna.

lower bands. Among the four resonances of the high band, only the $f_{4}$ resonance is shifted down greatly and the $f_{1}$ varies slightly as $L_{3}$ is increased, and in contrast, both $f_{1}$ and $f_{4}$ resonant modes are shifted up when $L_{4}$ is increased. This is due to the fact that in this case, the effective length of the long loop is altered.

Figure 7 shows the effect of the ground plane on the reflection coefficient, including the width and length of the ground plane. It is seen clearly that as the width of the ground plane is varied, a large change is produced in two operating bands. This is because the size of the resonant loop is altered and resonant frequency is deviated. However, when the length $L$ of the ground plane is increased from $113.5 \mathrm{~mm}$ to $153.5 \mathrm{~mm}$, a slight effect on the length of the reflection coefficient is produced in high-frequency bands, whereas a large variation is observed in low bands and the second resonance $f_{6}$ of the low band is shifted downward and the first resonance $f_{5}$ has no change, which is due to the fact that the first resonance is produced by using the high-pass circuit and the $0.5 \lambda$ loop mode generates the second resonance.

\section{Experimental Results}

The simulation results were verified by fabricating and testing a prototype of the proposed design with the dimensions provided in Figure 1. Figure 8(a) displays the photograph of the fabricated antenna. The performance of the antenna 

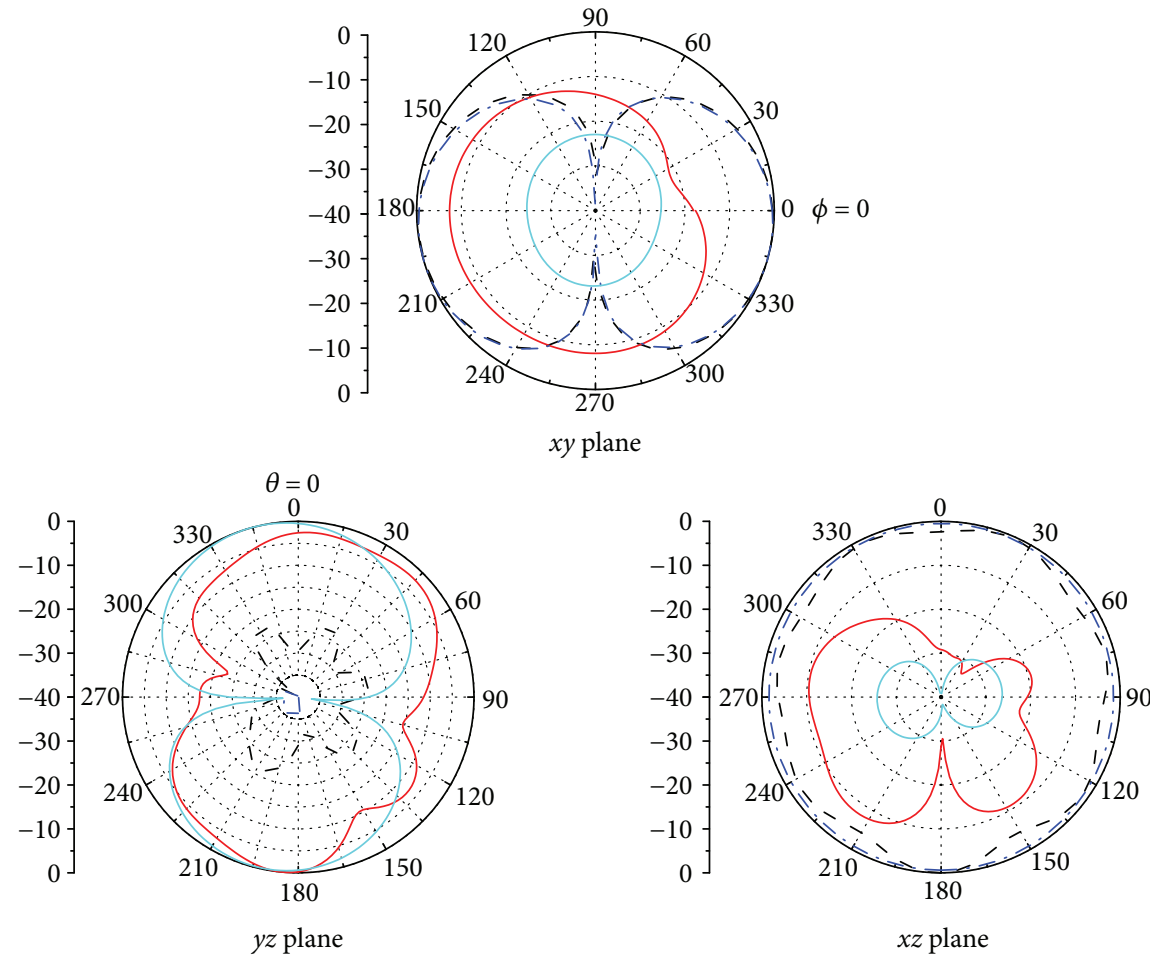

$\begin{array}{ll}--E_{\phi}(\text { Measured }) & -\cdots E_{\phi}(\text { Simulated }) \\ \left.-E_{\theta} \text { (Measured }\right) & -E_{\theta}(\text { Simulated })\end{array}$

(a)
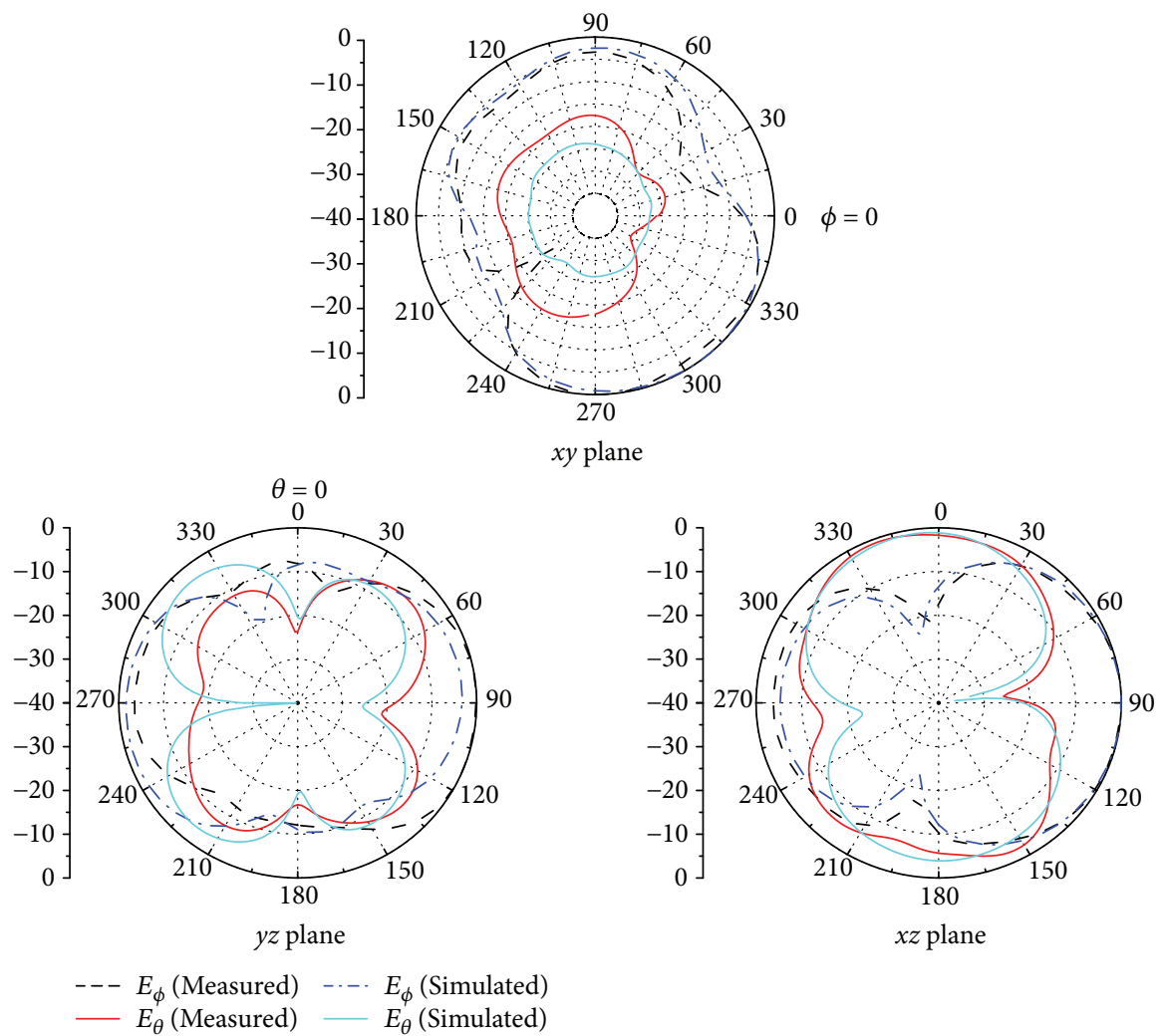

(b)

Figure 9: Normalized radiation patterns at two resonant frequencies: (a) $0.9 \mathrm{GHz}$ and (b) $1.8 \mathrm{GHz}$. 


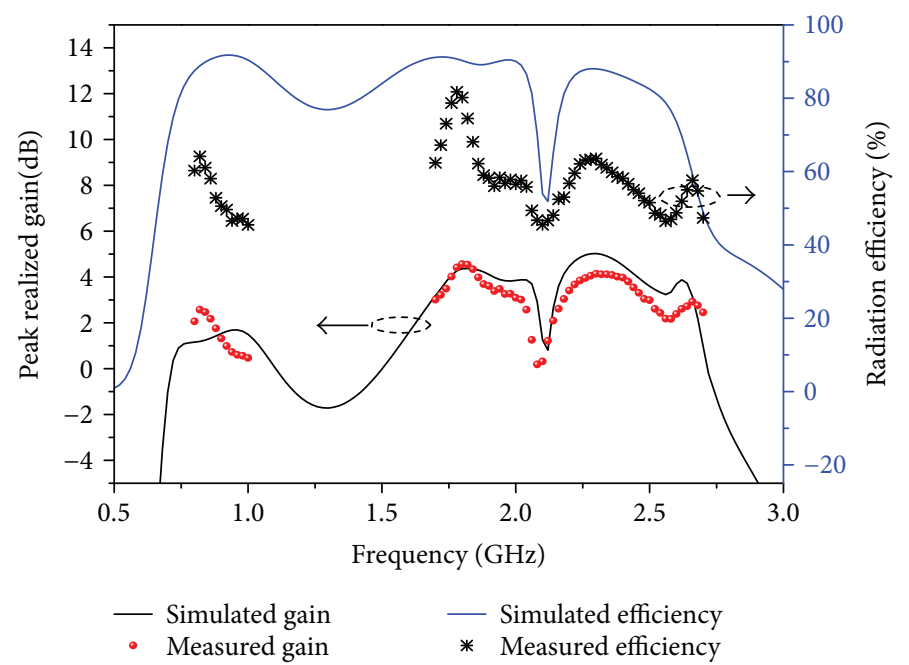

FIGURE 10: Simulated and measured peak realized gain and efficiency.

was measured by using a multiprobe antenna test chamber and Agilent N5230A series vector network analyzer.

3.1. Reflection Coefficient. Measured and simulated reflection coefficients for the proposed antenna are compared in Figure 8(b), where good agreement among the results is obvious. It is apparent from the figure that six resonant modes are excited successfully at the frequencies of $0.74 \mathrm{GHz}, 0.9 \mathrm{GHz}$, $1.73 \mathrm{GHz}, 2.1 \mathrm{GHz}, 2.3 \mathrm{GHz}$, and $2.64 \mathrm{GHz}$. It needs to be mentioned that a single broadband is achieved by intentionally merging the first two consecutive resonant modes of the lower band. Similarly, the merging of rest of the modes was done as well at the upper bands to attain a single broadband. From the measured reflection coefficient curves in Figure $8(\mathrm{~b})$, it is observed that the measured reflection coefficient is less than $-6 \mathrm{~dB}$ over the frequency ranges of about $0.7 \mathrm{GHz}-1.03 \mathrm{GHz}(38.2 \%)$ in the lower band and about $1.7 \mathrm{GHz}-2.69 \mathrm{GHz}$ in the upper band, which is wide enough to cover full LTE band services.

3.2. Radiation Pattern. Figure 9 presents the radiation patterns of the antenna. It is apparent from the figure that a dipole-like radiation pattern is obtained at $0.9 \mathrm{GHz}$. It means that in the azimuthal plane ( $x z$ plane), an omnidirectional radiation pattern is presented, which is in accordance with the pattern of the 0.5 -wavelength monopole mode. It is further observed that at higher frequencies, that is, $1.9 \mathrm{GHz}$ and $2.4 \mathrm{GHz}$, more variations and nulls appear in the patterns as compared to that at $0.9 \mathrm{GHz}$. The achieved radiation patterns are in similarity to those of several reported WWAN handset antennas.

3.3. Realized Gain and Efficiency. The simulated and measured gain and efficiency in the lower and upper bands are presented in Figure 10. For the lower band, the radiation efficiency is larger than $46.5 \%$ and the antenna gain varies from $0.56 \mathrm{dBi}$ to $2.57 \mathrm{dBi}$. For the upper band, the radiation efficiency is about $45.5 \%$ to $81.75 \%$ and the antenna gain varies from $0.19 \mathrm{dBi}$ to $4.56 \mathrm{dBi}$. It can also be noticed that the results deteriorate around the resonant frequency $2.1 \mathrm{GHz}$ of the centered band. The deterioration may be a consequence of the cancellation of a folded strip line with an opposite phase at the left side. Nevertheless, the achieved efficiency and gain values are still acceptable in practical mobile applications.

\section{Conclusion}

In this paper, a compact coupled-fed loop antenna with six resonant modes for mobile smartphones was proposed. The distinguishing attribute of the proposed antenna is that both front ends of the folded loop line are connected to system ground plane and the loop is fed capacitively by an unequal T-shaped strip to produce two wide operating bands. The dual resonance in the lower band, covering GSM850 and GSM900 bands, is provided by the half-wavelength loop mode in combination with a high-pass circuit. Other highorder modes of the entire loop line and the $0.5 \lambda$ mode of coupled loop at the left side are excited in the upper band to produce four resonances for DCS, PCS, UMTS, LTE2300, and LTE2500 operations. The measured results of the reflection coefficient and radiation characteristics of the proposed antenna signify its suitability for smartphone applications.

\section{Conflicts of Interest}

The authors declare that they have no conflicts of interest.

\section{Acknowledgments}

This work was supported by the Mainland Hong Kong, Macau, and Taiwan Science and Technology Cooperation Projects (2015DFT10170), the Science and Technology Research Project of Chongqing Municipal Education Committee under Grant no. KJ1600409, and the Doctoral Fund of Chongqing University of Posts and Telecommunications (A2015-08). 


\section{References}

[1] S. M. Ali, H. Gu, K. Wilson, and J. Warden, "Improved handset antenna performance via an electrically extended ground plane," ISRN Communications and Networking, vol. 2012, Article ID 621526, 7 pages, 2012.

[2] Y. W. Liang and H. M. Zhou, "Small-size seven-band WWAN/ LTE antenna with distributed LC resonant circuit for smartphone application," International Journal of Antennas and Propagation, vol. 2015, Article ID 630674, 9 pages, 2015.

[3] S. W. Lee, H. S. Jung, and Y. J. Sung, "A reconfigurable antenna for LTE/WWAN mobile handset applications," IEEE Antennas and Wireless Propagation Letters, vol. 14, pp. 48-51, 2015.

[4] W. H. Zong, X. M. Yang, X. Xiao et al., "A wideband antenna with circular and rectangular shaped slots for mobile phone applications," International Journal of Antennas and Propagation, vol. 2016, Article ID 2975425, 9 pages, 2016.

[5] W. S. Chen, C. M. Cheng, D. H. Lee, C. L. Ciou, W. S. Sin, and G. Y. Cai, "Small-size meandered loop antenna for WLAN dongle devices," International Journal of Antennas and Propagation, vol. 2014, Article ID 897654, 7 pages, 2014.

[6] K. Ishimiya, C.-Y. Chiu, and J.-i. Takada, "Multiband loop handset antenna with less ground clearance," IEEE Antennas and Wireless Propagation Letters, vol. 12, pp. 1444-1447, 2013.

[7] C.-W. Chiu, C.-H. Chang, and Y.-J. Chi, "A meandered loop antenna for LTE/WWAN operations in a smart phone," Progress In Electromagnetics Research C, vol. 16, pp. 147-160, 2010.

[8] P. Wang and Z. Cai, "Planar printed loop antenna with less no-ground space for hepta-band wireless wide area network/ long-term evolution mobile handset," Electronics Letters, vol. 52, no. 15, pp. 1284-1286, 2016.

[9] D. Wu, S. W. Cheung, and T. I. Yuk, "A compact and lowprofile loop antenna with multiband operation for ultra-thin smartphones," IEEE Transactions on Antennas and Propagation, vol. 63, no. 6, pp. 2745-2750, 2015.

[10] H. Xu, H. Wang, S. Gao et al., "A compact and low-profile loop antenna with six resonant modes for LTE smartphone," IEEE Transactions on Antennas and Propagation, vol. 64, no. 9, pp. 3743-3751, 2016.

[11] K.-L. Wong and Y.-C. Chen, "Small-size hybrid loop/open-slot antenna for the LTE smartphone," IEEE Transactions on Antennas and Propagation, vol. 63, no. 12, pp. 5837-5841, 2015.

[12] B. Lee, B. Kim, and S. Yang, "Enhanced loop structure of NFC antenna for mobile handset applications," International Journal of Antennas and Propagation, vol. 2014, Article ID 187029, 6 pages, 2014. 


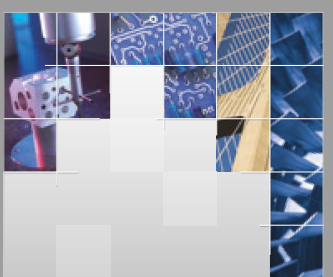

\section{Enfincering}
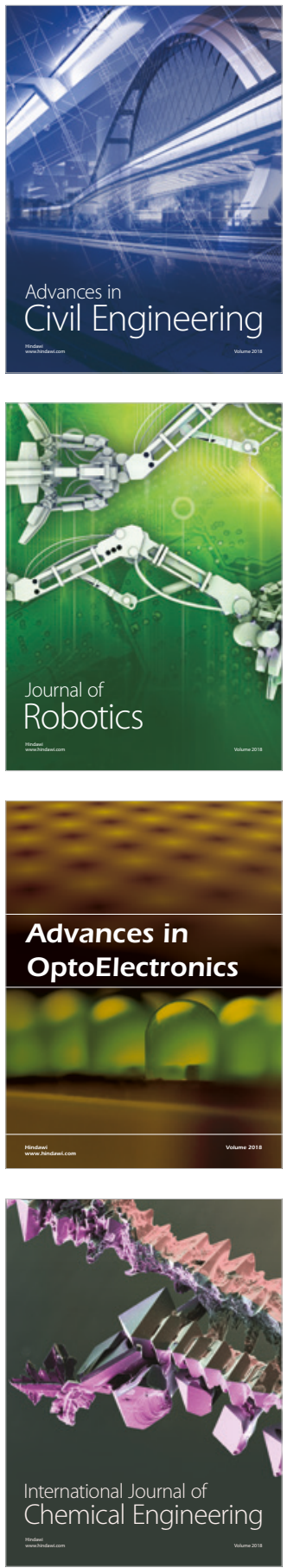

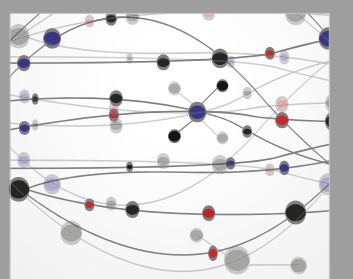

\section{Rotating \\ Machinery}

The Scientific World Journal

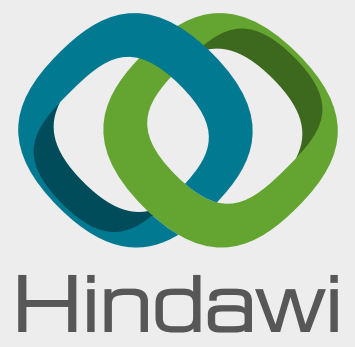

Submit your manuscripts at

www.hindawi.com
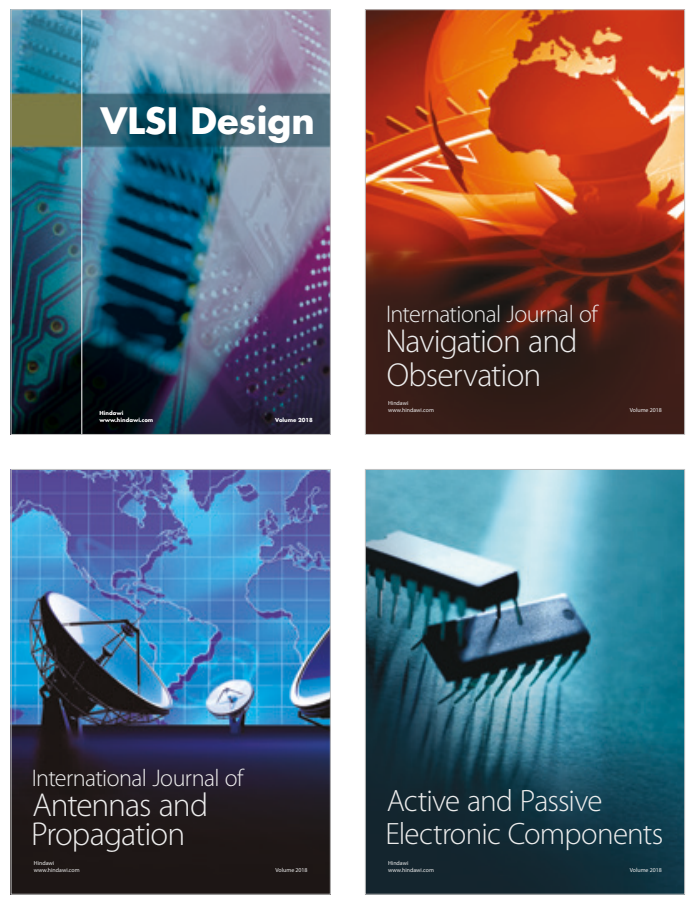
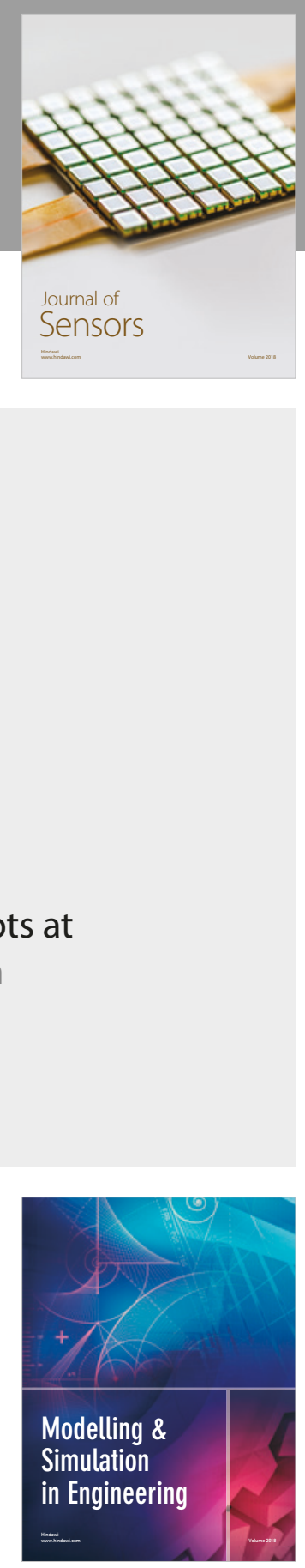

\section{Advances \\ Multimedia}
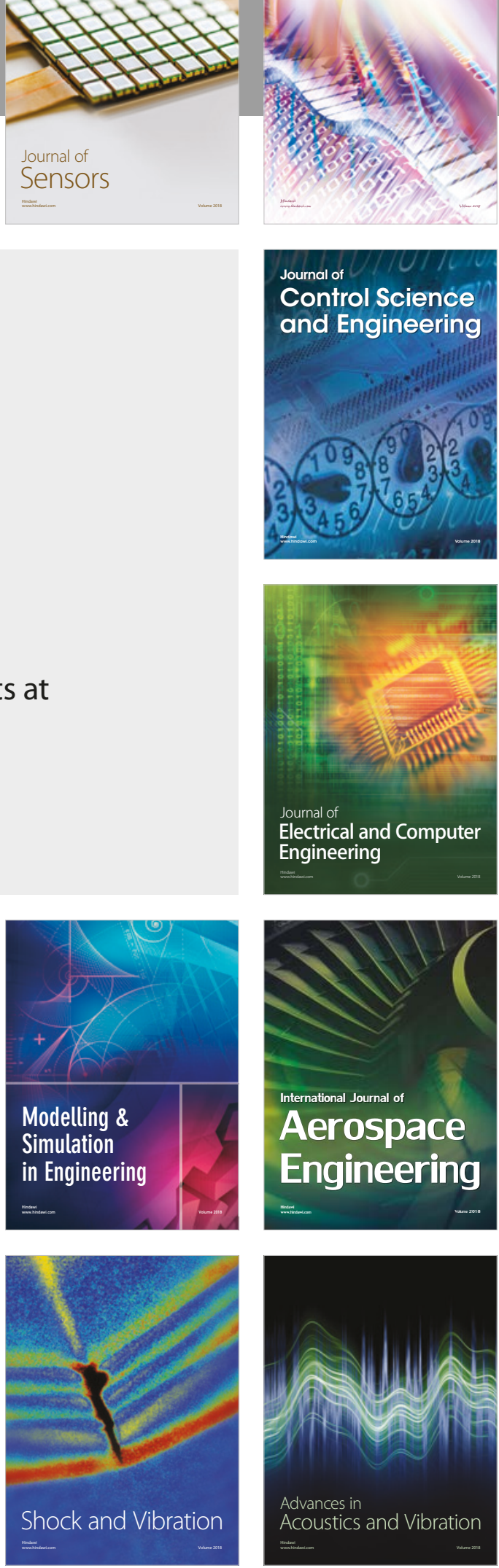